\title{
NEMA NU 4-2008 Performance Measurements of Two Commercial Small-Animal PET Scanners: ClearPET and rPET-1
}

\author{
Mario Cañadas, Miguel Embid, Eduardo Lage, Member, IEEE, Manuel Desco, \\ Juan José Vaquero, Senior Member, IEEE, and José Manuel Pérez, Member, IEEE
}

\begin{abstract}
In this work, we compare two commercial positron emission tomography (PET) scanners installed at CIEMAT (Madrid, Spain): the ClearPET and the rPET-1. These systems have significant geometrical differences, such as the axial field of view (110 mm on ClearPET versus $45.6 \mathrm{~mm}$ on $\mathrm{mPET}-1)$, the configuration of the detectors (whole ring on ClearPET versus one pair of planar blocks on rPET-1) and the use of an axial shift between ClearPET detector modules. We used an assessment procedure that fulfilled the recommendations of the National Electrical Manufacturers Association (NEMA) NU 4-2008 standard. The methodology includes studies of spatial resolution, sensitivity, scatter fraction, count losses and image quality. Our experiments showed a central spatial resolution of $1.5 \mathrm{~mm}$ (transaxial), 3.2 $\mathrm{mm}$ (axial) for the ClearPET and $1.5 \mathrm{~mm}$ (transaxial), $1.6 \mathrm{~mm}$ (axial) for the rPET-1, with a small variation across the transverse axis on both scanners $(\sim 1 \mathrm{~mm})$. The absolute sensitivity at the centre of the field of view was $4.7 \%$ for the ClearPET and $1.0 \%$ for the rPET-1. The peak noise equivalent counting rate for the mouse-sized phantom was $73.4 \mathrm{kcps}$ reached at $0.51 \mathrm{MBq} / \mathrm{mL}$ on the ClearPET and $29.2 \mathrm{kcps}$ at $1.35 \mathrm{MBq} / \mathrm{mL}$ on the rPET-1. The recovery coefficients measured using the image quality phantom ranged from 0.11 to 0.89 on the ClearPET and from 0.14 to 0.81 on the rPET-1. The overall performance shows that both the ClearPET and the rPET-1 systems are very suitable for preclinical research and imaging of small animals.
\end{abstract}

Index Terms-Image quality assessment, performance evaluation, PET, small-animal imagers.

\section{INTRODUCTION}

$\mathbf{S}$ MALL-ANIMAL positron emission tomography (PET) is becoming an essential imaging modality for preclinical research [1], [2] and in the search for new radio-pharmaceuticals [3]. At the same time, manufacturers of clinical PET systems use these scanners to test the technological aspects of new developments.

Manuscript received November 19, 2009; revised March 08, 2010 and July 01, 2010; accepted August 23, 2010. Date of publication November 09, 2010; date of current version February 09, 2011.

M. Cañadas, M. Embid, and J. M. Pérez are with the CIEMAT (Centro de Investigaciones Energéticas, Medioambientales y Tecnológicas), 28040 Madrid, Spain (e-mail: mario.canadas@ciemat.es).

E. Lage is with the Unidad de Medicina y Cirugía Experimental, Hospital General Universitario Gregorio Marañón, 28007 Madrid, Spain.

M. Desco is with the Departamento de Bioingeniería e Ingeniería Aeroespacial, Universidad Carlos III de Madrid, 28911 Madrid, Spain, and also with the Unidad de Medicina y Cirugía Experimental, Hospital General Universitario Gregorio Marañón, 28007 Madrid, Spain.

J. J. Vaquero is with the Departamento de Bioingeniería e Ingeniería Aeroespacial, Universidad Carlos III de Madrid, 28911 Madrid, Spain.
The comparison and evaluation of different scanners must be carried out following the same conditions, namely reconstruction protocol or the radioisotope used, which may significantly affect the results [4]. This is even more critical if the systems have different geometrical designs, such as the two scanners considered in this work. The ClearPET, manufactured by Raytest Isotopenmessgeraete $\mathrm{GmbH}$ (Mannheim, Germany), is composed of a rotating full ring of detectors with an axial field of view (FOV) of $11.0 \mathrm{~cm}$ and an axial-offset of $9.2 \mathrm{~mm}$ between each two adjacent detectors, whereas the rPET-1, manufactured by SEDECAL, S.A. (Madrid, Spain), has two rotating, planar block detectors and an axial FOV of $4.56 \mathrm{~cm}$. Both use pixelated crystals: a lutetium-yttrium orthosilicate (LYSO) and lutetium-yttrium aluminum perovskite (LuYAP) phoswich matrix with two layers in the ClearPET [5], and mixed lutetium silicate (MLS) with one layer in the rPET-1 [6]. The rPET-1 incorporates a coplanar CT (X-ray computed tomography) system so that it can work as a PET/CT scanner.

Although performance of both systems has been characterized previously [7], [8], the work presented here will compare them in the same conditions and following the recommendations of the National Electrical Manufacturers Association (NEMA) NU 4-2008 standard [9], created to evaluate small-animal PET systems [10]-[13]. The main interest of this work is to assess the influence of the design differences between the two scanners, specifically the axial-shifted detectors [14] and the planar versus ring configuration.

\section{Materials AND MethodS}

All the measurements were carried out on the scanners located at CIEMAT (Centro de Investigaciones Energéticas, Medioambientales y Tecnológicas), Madrid (Spain). Table I shows a summary of the geometrical and physical characteristics of both systems. It is important to remark that the rPET-1 system is composed of only two block detectors, while other available versions of rPET systems have four blocks [13]. This will essentially affect the transaxial FOV and the global sensitivity of the two-block system, which is close to one half of the four-block version scanner. While the detector modules in the ClearPET can change the inner diameter from $13.5 \mathrm{~cm}$ to $22 \mathrm{~cm}$ for mouse/rat or primate imaging, this work focuses on the small-diameter configuration. Fig. 1 shows the gantry and detector modules of both scanners.

While the scintillator materials used in the scanners are different, LYSO and MLS crystals have similar properties in terms 
TABLE I

CHARACTERISTICS OF THE CLEARPET AND THE RPET-1 SYSTEMS

\begin{tabular}{|c|c|c|}
\hline Detector & ClearPET & rPET-1 \\
\hline Detector ring diameter $(\mathrm{mm})$ & $135 / 220^{a}$ & 140 \\
\hline Number of detector modules & 20 & 2 \\
\hline $\begin{array}{l}\text { Number of PMTs } \\
\text { (photomultiplier tubes) }\end{array}$ & 80 & 2 \\
\hline Total PMT sensitive area $\left(\mathrm{cm}^{2}\right)$ & 262.1 & 48.0 \\
\hline PMT type & $\begin{array}{c}\text { Position-sensitive, } \\
\text { Hamamatsu } \\
\text { R7600-M64 }\end{array}$ & $\begin{array}{c}\text { Position-sensitive, } \\
\text { Hamamatsu } \\
\text { H8500 }\end{array}$ \\
\hline $\begin{array}{l}\text { Layers of crystals, radial } \\
\text { direction }\end{array}$ & 2 & 1 \\
\hline Crystal size $\left(\mathrm{mm}^{3}\right)$ & $2 \times 2 \times 10^{b}$ & $1.4 \times 1.4 \times 12$ \\
\hline Crystal pitch (mm) & 2.3 & 1.5 \\
\hline Crystal material & LYSO / LuYAP ${ }^{c}$ & MLS \\
\hline Total scintillator volume $\left(\mathrm{cm}^{3}\right)$ & 409.6 & 42.3 \\
\hline \multicolumn{3}{|l|}{ System } \\
\hline Maximum transaxial FOV (mm) & $94 / 144^{a}$ & 45.6 \\
\hline Axial FOV (mm) & 110 & 45.6 \\
\hline Rotating gantry & Yes & Yes \\
\hline Coincidence window (ns) & 12 & 3.8 \\
\hline Energy windows (keV) & $\begin{array}{l}100-750 \\
250-750 \\
400-750\end{array}$ & $\begin{array}{l}100-700 \\
250-650 \\
400-700\end{array}$ \\
\hline Image pixel size (mm) & $1.15 / 0.57 / 0.38$ & $0.77 / 0.46 / 0.27$ \\
\hline Slice thickness (mm) & 1.15 & 0.77 \\
\hline Reconstruction methods & $\begin{array}{c}\text { 2D/3D FBP } \\
2 \mathrm{D} / 3 \mathrm{D} \mathrm{OSEM}^{\mathrm{c}}\end{array}$ & $\begin{array}{l}\text { 2D/3D FBP } \\
\text { 2D/3D OSEM }\end{array}$ \\
\hline
\end{tabular}

a Two adjustable detector diameters for rodent whole body or primate brain studies.

b Two radial layers of $10 \mathrm{~mm}$.

"Front layer: LYSO, back layer: LuYAP.

'Filtered back projection.

"Ordered subsets expectation maximization [15].

of density $\left(7.15-7.4 \mathrm{~g} / \mathrm{cm}^{3}\right)$, wavelength of maximum emission $(420 \mathrm{~nm})$ and scintillation decay time (40 ns). LuYAP differs in the decay time and wavelength of maximum emission (20 ns, $375 \mathrm{~nm}$ ), although it has a similar density.

Data processing is significantly different between these systems. The data acquisition system of the ClearPET [16] consists of 20 detector cassettes, each comprising 4 PMTs and electronics for trigger, shaping, data-digitization (free-running analog-to-digital converters, ADC, at $40 \mathrm{MHz}$ sampling rate and 12-bit resolution) and transmission. These cassettes transfer their data via fiber optics to five preprocessing PCs (four cassettes per PC) which provide list-mode files with the information of each single photon detected. From there, a Giga-bit Ethernet connection leads the preprocessed data to a master PC, which also controls the acquisition and hosts the interface for the user. The maximum count rate this system can process is roughly 3 Mcounts. $\mathrm{s}^{-1}$ (single photons). Coincidence sorting is performed in the master computer after acquisition.
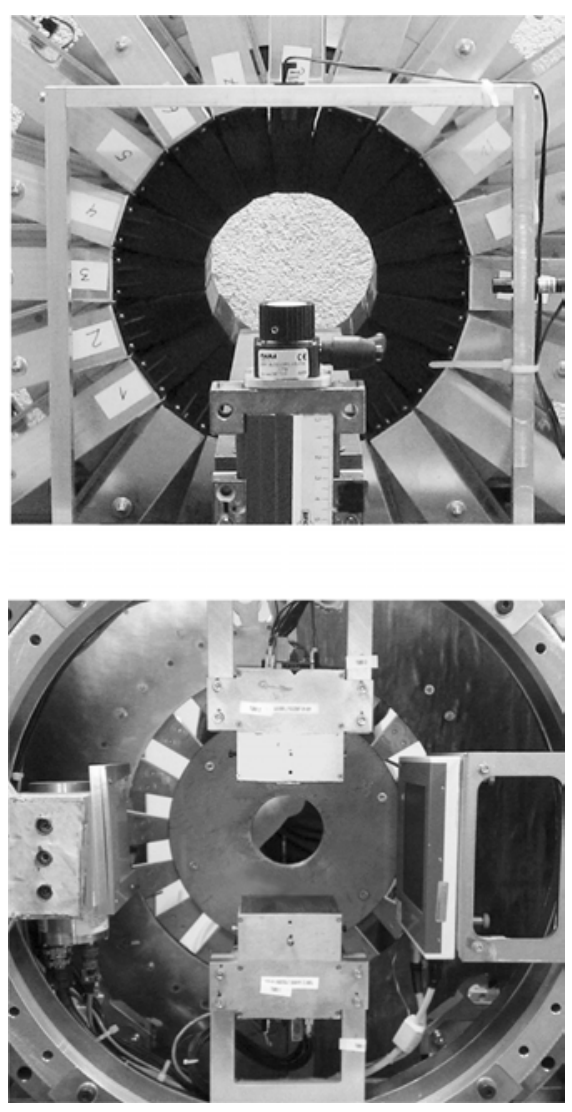

Fig. 1. Detector modules of the ClearPET (top) and rPET-1 (bottom). Note that the rPET-1 image includes the X-ray CT system (horizontal orientation) which is mounted on the same gantry as the PET modules (vertical orientation).

In contrast, the rPET-1 uses a processing scheme consisting of hardware-based coincidence detection. Read-out electronics of each detector are integrated in a compact printed circuit board (PCB) stack directly attached to the PMT sockets. These PCBs include a charge division circuit and amplification stages for the position and timing signals. The data acquisition system consists of an arbiter module in charge of coincidence detection (based on timing signals from the detectors) and generation of trigger signals, two ADC cards for data digitization, and a 32-bit digital I/O peripheral component interconnect (PCI) card for data transmission to the acquisition computer. After the detection of a valid event, trigger signals are transmitted to the ADC modules to start the digitization of the position signals. These signals are integrated for a $150-\mathrm{ns}$ period, digitized and sent to the PC in order of arrival. The complete processing of each pair of photons takes $1.8 \mu \mathrm{s}$, leading to a maximum processing throughput of roughly $500 \mathrm{kcounts} \cdot \mathrm{s}^{-1}$ (coincidences). During scans, the acquisition computer receives the data from the PCI interface and processes them in real time to generate list-mode files containing the coincidence events information.

The methodology used to evaluate the performance of both scanners follows the recommendations of the NEMA NU 4-2008 standard [9]. This protocol was recently approved by an international committee composed of university researchers and manufacturers of small-animal PET systems, including the two considered here. 
This work includes studies of:

- Spatial resolution;

- Sensitivity;

- Scatter fraction, count losses and random coincidence measurements (toward mouse imaging);

- Image quality.

In addition, a micro Derenzo ${ }^{22} \mathrm{Na}$ sealed phantom (described in Section II.E of this paper) was scanned and reconstructed on both systems. The acquisition of a Derenzo image, although not included in the NEMA protocol, is a common way to illustrate the spatial resolution of a scanner.

\section{A. Spatial Resolution}

A $0.25-\mathrm{mm}$ diameter, $0.8-\mathrm{MBq}{ }^{22} \mathrm{Na}$ point source was scanned at equivalent positions in both systems. The measurements were taken in the central slice of the axial FOV, at the following radial distances from the geometrical centre: $0 \mathrm{~mm}, 5$ $\mathrm{mm}, 10 \mathrm{~mm}, 15 \mathrm{~mm}, 20 \mathrm{~mm}$ and $25 \mathrm{~mm}$. Resolution at the same transaxial points was also evaluated at $1 / 4$ axial FOV. Acquisition at $0 \mathrm{~mm}$ is not included in the NEMA document, although the results at this location have been presented in previous characterizations of both systems [7], [8]. We added this position to measure the spatial resolution at the centre of the FOV, but this is not shown in the table presenting the NEMA values. The NEMA document also recommends a measurement at $25 \mathrm{~mm}$ from the centre; this location is out of the transaxial FOV of the rPET-1. More than $10^{5}$ prompt counts were acquired per measurement, and analytic image reconstruction algorithms with no-smoothing filters were applied on both scanners. In the ClearPET, a 3D Filtered Back Projection algorithm (the FBP3DRP algorithm from the STIR package) [17], [18] was used with an image pixel size of $0.38 \mathrm{~mm}$ and a slice thickness of $1.15 \mathrm{~mm}$. The FBP3DRP algorithm was also used on the rPET-1 (0.27-mm pixel size, 0.77-mm slice thickness).

We report the width of the reconstructed image point spread functions (PSF), defining the width as its full width at half-maximum amplitude (FWHM), and the full width at tenth-maximum amplitude (FWTM). The response function is formed by summing all one-dimensional profiles that are parallel to the direction of measurement (radial, tangential or axial) and within two times the FWHM of the orthogonal directions. The fitting method used to assess each FWHM (and FWTM) fulfills the NEMA NU 4-2008 protocol recommendations.

\section{B. Sensitivity}

Sensitivity is expressed as the rate in counts per second (cps) at which true coincidence events are detected for a given source intensity and branching ratio; absolute sensitivity is the fraction of positron annihilation events detected as true coincidence events. The same ${ }^{22} \mathrm{Na}$ point source described previously was scanned in small position increments along the entire axial FOV. For each scanner, the considered increment is equal to its reconstructed plane thickness.

The variables $S_{\text {tot }}, S M_{\text {tot }}$, and $S R_{\text {tot }}$ represent the total, total mouse $(7 \mathrm{~cm})$ and total rat $(15 \mathrm{~cm})$ axial length sensitivities. Their values are also reported as the absolute sensitivities $\left(S_{A, \text { tot }}, S M_{A, \text { tot }}, S R_{A, \text { tot }}\right)$ and as the sensitivity profiles by plotting the absolute sensitivity for each slice. These variables are strongly dependent on the energy window considered; the NEMA values are presented using the energy window recommended for routine studies (250-750 keV for the ClearPET and 250-650 keV for the rPET-1). In addition to these results, we measured a sensitivity profile for the widest window available on each system (100-750 keV for the ClearPET, and 100-700 $\mathrm{keV}$ for the rPET-1). Both manufacturers recommend the widest window for low-dose studies.

\section{Scatter Fraction and Count Rate Measurements}

The NEMA document specifies three test phantom designs simulating mouse, rat and primate sizes. This work focuses on mouse studies and the measurements presented are related to the mouse-sized phantom. This phantom is a solid cylinder composed of high-density polyethylene (density $0.95 \mathrm{~g} / \mathrm{cm}^{3}$ ) $70 \mathrm{~mm}$ long and $25 \mathrm{~mm}$ in diameter. A cylindrical hole $(3.2 \mathrm{~mm}$ in diameter) is drilled parallel to the central axis at a radial distance of $10 \mathrm{~mm}$. A line source made of flexible tubing and filled to 60 $\mathrm{mm}$ with a known activity concentration of ${ }^{18} \mathrm{~F}$ is inserted into the hole.

Count rate and count loss measurements show the effects of system dead time and the presence of random coincidence events at different levels of source activity. These studies start with high activity in the phantom, exceeding the expected maximum count rate on both scanners.

We followed the procedure to classify random plus scattered counts for each slice $i$ and frame $j\left(C_{r+s, i, j}\right)$ as defined in Section 4 of the NEMA document. Counts were obtained from the sinogram of each axial slice formed after single-slice rebinning (SSRB) [19]. A sum projection was produced by adding all projections in each slice and each frame. Then, $C_{r+s, i, j}$ was computed as the sum of all counts outside a 14-mm centered strip plus the counts under the profile peak. The scattered and random counts under the peak were estimated by a linear interpolation between the left and right border of the 14-mm strip. The true rate $R_{t, i, j}$ was calculated as shown in (1), where $C_{\mathrm{TOT}, i, j}$ is the sum of the total counts, and $T_{\mathrm{acq}, j}$ is the acquisition time of frame $j$.

$$
R_{t, i, j}=\frac{C_{\mathrm{TOT}, i, j}-C_{r+s, i, j}}{T_{\mathrm{acq}, j}}
$$

The noise equivalent counting (NEC) rate describes the equivalent coincidence counting rate that would have the same noise properties as the net true counting rate, corrected for random and scattered events [20]; and is defined as:

$$
R_{\mathrm{NEC}, i, j}=\frac{R_{t, i, j}^{2}}{R_{\mathrm{TOT}, i, j}}
$$

The system true event rate $R_{t, j}$ and the system noise equivalent count rate $R_{\mathrm{NEC}, j}$ are calculated as the sum of $R_{t, i, j}$ and $R_{\mathrm{NEC}, i, j}$ over all slices $i$.

Both systems have natural radioactivity in the crystal due to the presence of ${ }^{176} \mathrm{Lu}$. This isotope emits $\beta^{-}$particles with an average energy of $420 \mathrm{keV}$, together with $\gamma$-photons of 307 , 202 , and $88 \mathrm{keV}$, all of which increase the single count rate of the detectors and may create events that can be recognized as 
true coincidences [21]. For the rPET-1, the intrinsic true event rate is $7.7 \mathrm{cps}$ using the energy window of $250-650 \mathrm{keV}$. The ClearPET reaches an intrinsic true event rate of $560 \mathrm{cps}$ for the $250-750 \mathrm{keV}$ energy window. These values were included in the assessment of the scatter fraction and count rates [22]; we obtained the intrinsic true event rate for each slice, $R_{\text {int }, i}$, from an acquisition using the phantom without activity.

Acquisitions $j^{\prime}$ are defined as those with random events below $1 \%$ of the true events. At such a low count rate, the randoms are negligible and $R_{r+s, i, j^{\prime}}$ becomes $R_{s, i, j^{\prime}}$ plus $R_{\text {int }, i}$. As $R_{\text {int }, i}$ is known, $R_{s, i, j^{\prime}}$ can be assessed at low activity. We used it to calculate the scatter fraction for slice $i\left(\mathrm{SF}_{i}\right)$ as shown in (3).

$$
\mathrm{SF}_{i}=\frac{\sum_{j^{\prime}} R_{s, i, j^{\prime}}}{\sum_{j^{\prime}}\left(R_{t, i, j^{\prime}}+R_{s, i, j^{\prime}}\right)}
$$

The system scatter fraction (SF) is computed as the weighted average of the $\mathrm{SF}_{i}$ values as follows:

$$
\mathrm{SF}=\frac{\sum_{i} \sum_{j^{\prime}} R_{s, i, j^{\prime}}}{\sum_{i} \sum_{j^{\prime}}\left(R_{t, i, j^{\prime}}+R_{s, i, j^{\prime}}\right)}
$$

The NEC rate obtained from (2) is valid for acquisitions without direct random event subtraction, as in the studies considered here. In this case the random event rate for acquisition $j$ and slice $i, R_{r, i, j}$, is estimated using the information of $\mathrm{SF}_{i}$ as shown in (5). The system random event rate, $R_{r, j}$, is computed as the sum of $R_{r, i, j}$ over all slices.

$$
R_{r, i, j}=R_{\mathrm{TOT}, i, j}-\frac{R_{t, i, j}}{1-\mathrm{SF}_{i}}
$$

The measurements were performed using the energy window recommended for routine studies in each scanner (250-750 $\mathrm{keV}$ for the ClearPET and 250-650 keV for the rPET-1). The phantom was centered in the FOV and the acquisition frames were selected as follows: frames of 300 seconds starting every 1200 seconds for the ClearPET and frames of 800 seconds starting every 1600 seconds for the rPET-1. The initial activity concentration was $1.25 \mathrm{MBq} / \mathrm{mL}$ (43 MBq total activity, ${ }^{18} \mathrm{~F}$ ) for the ClearPET and $1.61 \mathrm{MBq} / \mathrm{mL}\left(55.4 \mathrm{MBq},{ }^{18} \mathrm{~F}\right)$ for the rPET-1. The low activity acquisitions $\left(j^{\prime}\right)$ were those with a total activity in the phantom below $5 \mathrm{MBq}$.

\section{Image Quality}

Image quality studies were performed using the phantom described in the NEMA NU 4-2008 protocol. The phantom is a polymethylmethacrylate cylinder measuring $50 \mathrm{~mm}$ in length and $30 \mathrm{~mm}$ in diameter. It consists of three parts: one is composed of five fillable rods used to measure the noise and recovery coefficients as a function of rod diameter; one is a large uniform region connected to the rods allowing the uniformity to be measured; and the third is composed of two cold region chambers (filled with water and air) that are used to quantify the spillover ratio.

The recovery coefficients (RC, measured activity concentration divided by the actual activity concentration), percent standard deviation (\%STD, standard deviation divided by mean multiplied by 100 percent), and spillover ratio (SOR, activity concentration in the cold regions relative to the mean activity concentration in the hot background) were evaluated as described in the NEMA document.

A 20-minute acquisition was performed after filling the phantom with an ${ }^{18} \mathrm{~F}$ solution with an initial activity concentration of $0.194 \mathrm{MBq} / \mathrm{mL}$ (3.78 MBq total activity) for ClearPET and $0.185 \mathrm{MBq} / \mathrm{mL}$ (3.61 MBq) for rPET-1. The studies were performed using an energy window of 250-750 $\mathrm{keV}$ for the ClearPET and of 250-650 keV for the rPET-1. Iterative algorithms were used in both scanners according to the manufacturers' recommendations. In the ClearPET, a 3D ordered-subsets expectation maximization (OSEM) method [15], [18] was applied with 2 iterations and 10 subsets. For the data acquired in the rPET-1, a 3D-OSEM algorithm [23] was applied using a single iteration and 50 subsets. Correction for random events was only applied in the ClearPET. The rPET-1 system does not provide correction for randoms; however, at the usual activities in mouse studies $(15-20 \mathrm{MBq})$ the randoms fraction is below $5 \%$ of the total coincidences. The values presented in the image quality test were obtained without applying attenuation or scatter corrections.

\section{E. Derenzo Phantom Images}

Acquisition of a Derenzo image is an intuitive way to illustrate the spatial resolution and assess the overall image quality of a scanner. Nevertheless, it is important to remark that this study is not part of the NEMA NU 4-2008 document. We used $\mathrm{a}^{22} \mathrm{Na}$ sealed phantom (I.S.E. srl, Pisa, Italy) presenting a total activity of $0.76 \mathrm{MBq}$. It is composed of 20 rods measuring 1.2 $\mathrm{mm}$ in diameter, 14 rods measuring $1.5 \mathrm{~mm}, 9$ rods measuring $2.0 \mathrm{~mm}, 6$ rods measuring $2.5 \mathrm{~mm}$ and 3 rods measuring 3.0 $\mathrm{mm}$. The distance between rods of equal diameter is twice the diameter of the rod, centre to centre. The external dimensions of the phantom are $43 \mathrm{~mm}$ in length by $40 \mathrm{~mm}$ in diameter.

Acquisitions of one hour for the ClearPET, and five hours for the rPET-1, were performed in order to accumulate a similar number of coincidences in each system (approximately 80 million coincidences). The energy windows and reconstruction methods were the same as those for the image quality studies.

\section{RESULTS}

\section{A. Spatial Resolution}

The average one-dimensional FWHM over the three axes at the centre of the FOV was $2.1 \mathrm{~mm}$ (FWTM: $4.3 \mathrm{~mm}$ ) in the ClearPET and $1.6 \mathrm{~mm}$ (FWTM: $2.8 \mathrm{~mm}$ ) in the rPET-1. Resolution over the same transaxial points was also assessed at $1 / 4$ axial FOV, and no significant differences were found from the values shown at the axial centre of the FOV. Tables II and III 
TABLE II

SPATIAL RESOlUtion OF THE CLEARPET. FWHM (FWTM) IN MM. IMAGE PIXEL SIZE: 0.38 MM

\begin{tabular}{lccccc}
\hline \hline $\begin{array}{l}\text { At axial } \\
\text { centre }\end{array}$ & $\mathbf{5} \mathbf{~ m m}$ & $\mathbf{1 0} \mathbf{~ m m}$ & $\mathbf{1 5} \mathbf{~ m m}$ & $\mathbf{2 0} \mathbf{~ m m}$ & $\mathbf{2 5} \mathbf{~ m m}$ \\
\hline Radial & $1.9(3.8)$ & $1.8(3.4)$ & $2.0(3.6)$ & $2.6(4.5)$ & $2.5(4.3)$ \\
Tangential & $2.0(4.2)$ & $2.3(5.0)$ & $2.4(5.5)$ & $2.3(4.5)$ & $2.4(5.7)$ \\
Axial & $3.2(6.0)$ & $3.2(6.0)$ & $3.2(6.0)$ & $3.2(5.9)$ & $3.2(6.0)$ \\
\hline At 1/4 & & & & & \\
axial FOV & & & & & \\
\hline Radial & $2.2(4.0)$ & $1.9(3.7)$ & $2.0(3.8)$ & $2.4(3.9)$ & $2.5(4.2)$ \\
Tangential & $2.0(3.9)$ & $2.1(5.0)$ & $2.3(5.2)$ & $2.3(5.7)$ & $2.4(5.6)$ \\
Axial & $3.2(5.9)$ & $3.2(5.9)$ & $3.2(5.8)$ & $3.2(5.8)$ & $3.2(5.8)$ \\
\hline \hline
\end{tabular}

TABLE III

SPATIAl Resolution OF THE RPET-1. FWHM (FWTM) IN MM. IMAGE PiXeL SIZE: $0.27 \mathrm{MM}$

\begin{tabular}{lllllll}
\hline \hline $\begin{array}{l}\text { At axial } \\
\text { centre }\end{array}$ & $\mathbf{5} \mathbf{~ m m}$ & $\mathbf{1 0} \mathbf{~ m m}$ & $\mathbf{1 5} \mathbf{~} \mathbf{m}$ & $\mathbf{2 0} \mathbf{~ m m}$ & $\mathbf{2 5} \mathbf{m m}^{\mathrm{a}}$ \\
\hline Radial & $1.4(2.5)$ & $1.3(2.3)$ & $1.1(2.1)$ & $1.1(1.9)$ & - \\
Tangential & $1.6(3.0)$ & $1.8(3.3)$ & $2.1(3.9)$ & $2.4(4.3)$ & - \\
Axial & $1.8(3.2)$ & $2.1(3.8)$ & $2.3(4.1)$ & $2.7(4.8)$ & - \\
\hline At 1/4 & & & & & & \\
axial FOV & & & & & & \\
\hline Radial & $1.5(2.8)$ & $1.5(2.7)$ & $1.3(2.4)$ & $1.4(2.6)$ & - \\
Tangential & $1.6(3.0)$ & $1.7(3.0)$ & $1.9(3.8)$ & $1.7(3.2)$ & - \\
Axial & $1.5(2.8)$ & $1.8(3.3)$ & $2.1(3.9)$ & $2.4(4.7)$ & - \\
\hline \hline
\end{tabular}

${ }^{a}$ The measurement point is outside the transaxial FOV

show the results of the spatial resolution obtained in both systems.

The volumetric resolution at the centre was $7.6 \mathrm{~mm}^{3}$ on the ClearPET and $3.8 \mathrm{~mm}^{3}$ on the rPET-1. Fig. 2 presents the spatial resolution (FWHM) obtained at the axial centre of FOV. ClearPET values of axial FWHM are $1.5 \mathrm{~mm}$ lower than rPET-1 at central positions; rPET-1 shows a small $(\sim 1 \mathrm{~mm})$ degradation of the axial resolution along the transaxial axis that is not observed in the ClearPET. Axial resolution is strongly dependent on slice thickness; its value $(1.15 \mathrm{~mm}$ in the ClearPET and $0.77 \mathrm{~mm}$ in the rPET-1) is half of the axial crystal pitch in each scanner.

Both scanners showed a variation of approximately $1 \mathrm{~mm}$ of the FWHM across the transaxial axis in the tangential direction. For these points, the rPET- 1 shows an improvement of $0.5 \mathrm{~mm}$ in the radial direction.

\section{B. Sensitivity}

The absolute sensitivity obtained at the centre of the FOV was $4.7 \%$ in ClearPET and $1.0 \%$ in rPET-1 for the widest energy window on each system. Total length $\left(S_{\text {tot }}\right)$ and mouse length $\left(S M_{\text {tot }}\right)$ sensitivity values are shown in Table IV for the energy windows recommended by both manufacturers for routine studies (250-750 keV for the ClearPET and $250-650 \mathrm{keV}$ for the rPET-1). $S_{\text {tot }}$ and $S M_{\text {tot }}$ values are identical in the rPET-1,
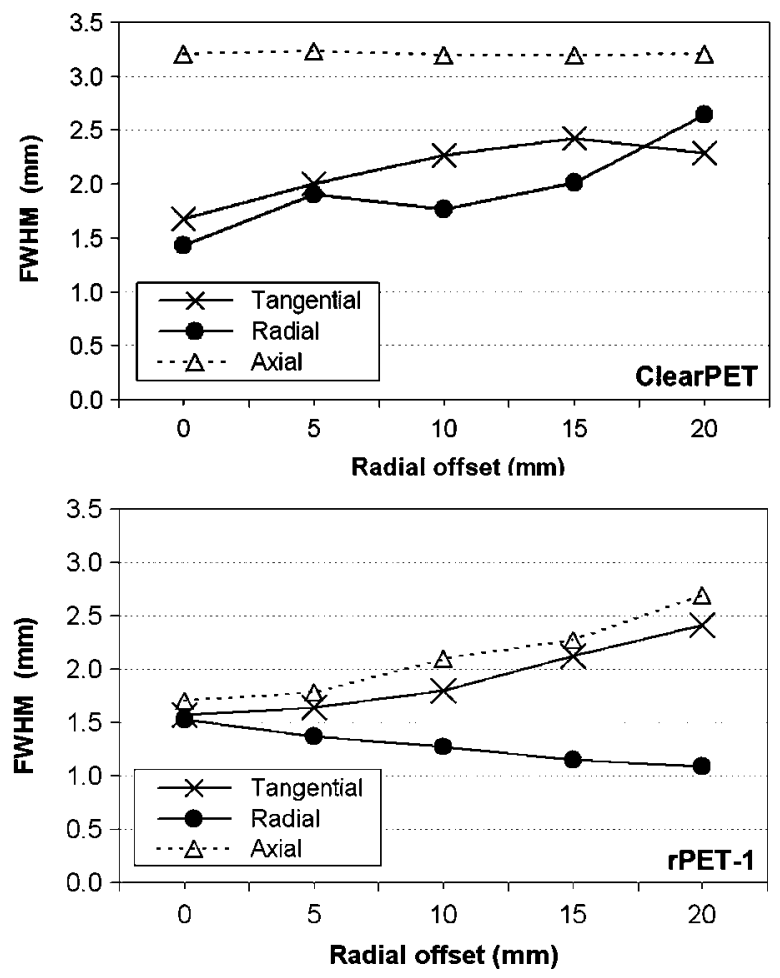

(b)

Fig. 2. ClearPET (a) and rPET-1 (b) spatial resolution (FWHM) in all directions as a function of the radial distance from the centre. Results are shown for the source positioned at the centre of the axial FOV.

TABLE IV

NEMA SENSITIVITY RESULTS FOR THE CLEARPET AND THE RPET-1

\begin{tabular}{ccc}
\hline \hline & ClearPET & rPET-1 \\
\hline $\mathrm{S}_{A, t o t}(\%)$ & 1.87 & 0.46 \\
$\mathrm{SM}_{A, t o t}(\%)$ & 2.32 & 0.46 \\
$\mathrm{~S}_{\text {tot }}(\mathrm{kcps} / \mathrm{MBq})$ & 16.98 & 4.18 \\
$\mathrm{SM}_{\text {tor }}(\mathrm{kcps} / \mathrm{MBq})$ & 21.08 & 4.18 \\
\hline \hline
\end{tabular}

because the axial length of the system is lower than the length considered for mouse studies $(7 \mathrm{~cm})$.

Fig. 3 plots the NEMA absolute sensitivity profile as a function of the axial distance from the centre. An important characteristic of the ClearPET is the 9.2- $\mathrm{mm}$ axial displacement between each two adjacent detectors which enlarges the axial FOV; this produces a less homogeneous sensitivity profile than the rPET-1. Therefore, a specific normalization procedure is required to compensate for the lack of homogeneity across the axial axis [24]. This is also the cause of the absolute sensitivity peaks (above $5 \%$, Fig. 3) which ClearPET reaches at $\pm 5 \mathrm{~mm}$ from the axial centre of FOV.

\section{Scatter Fraction and Count Rate Measurements}

The scatter fractions obtained on each system were $31.0 \%$ for the ClearPET and $24.2 \%$ for the rPET- 1 with the mouse-sized phantom.

Count rate results are presented for the mouse-sized phantom. Fig. 4 shows the true, random, scattered, NEC and total count rate (kcps) as a function of the average effective activity concentration $(\mathrm{MBq} / \mathrm{mL})$. The peak true count rate $\left(R_{t, \text { pgak }}\right)$, the 


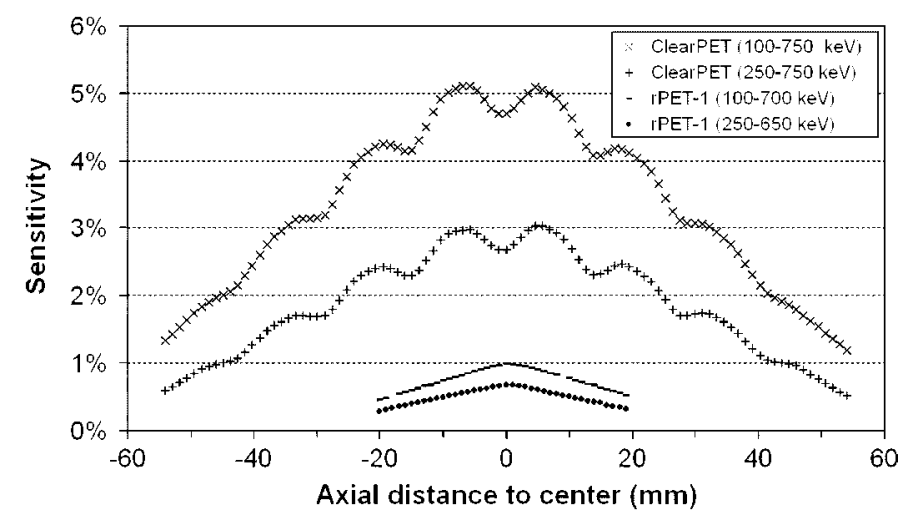

Fig. 3. ClearPET and rPET-1 absolute sensitivity profiles for different energy windows.
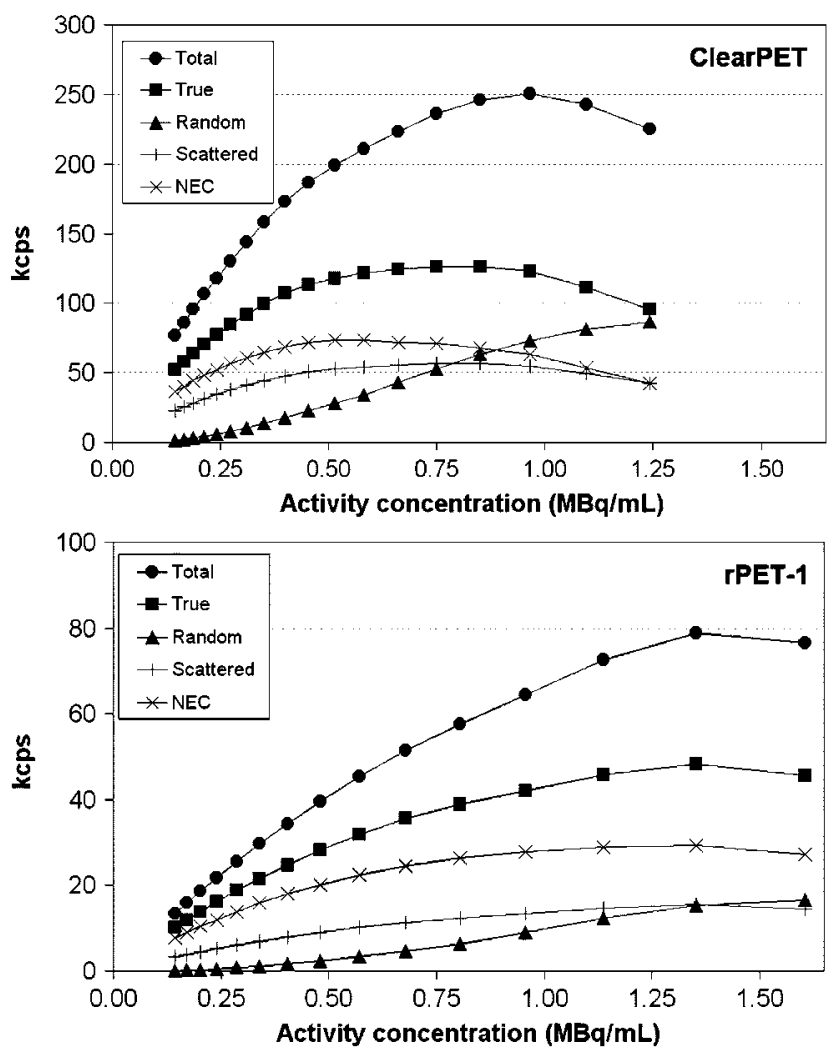

Fig. 4. ClearPET (a) and rPET-1 (b) total, true, random, scattered and NEC count rates as a function of the average activity in the mouse-sized phantom.

TABLE V

COUNT RATE Results FOR THE CLEARPET AND RPET-1

\begin{tabular}{ccc}
\hline \hline & ClearPET & rPET-1 \\
\hline $\mathrm{R}_{\text {flues, petlk }}(\mathrm{kcps})$ & 126.0 & 78.9 \\
$\mathrm{R}_{\text {NEC, peak }}(\mathrm{kcps})$ & 73.4 & 29.2 \\
$\mathrm{a}_{\text {trites, peak }}(\mathrm{MBq} / \mathrm{mL})$ & 0.75 & 1.35 \\
$\mathrm{a}_{\text {NEC, peuk }}(\mathrm{MBq} / \mathrm{mL})$ & 0.51 & 1.35 \\
\hline \hline
\end{tabular}

peak NEC rate $\left(R_{\mathrm{NEC}, \text { peak }}\right)$, and the activity concentration at which they are reached $\left(a_{t, \text { peak }}, a_{\mathrm{NEC}, \text { peak }}\right)$ were also assessed for both scanners and are summarized in Table V.

(b)
TABLE VI

IMAGE RECOVERY COEFFICIENTS AND \%STD ON BOTH SCANNERS

\begin{tabular}{|c|c|c|c|c|c|}
\hline Rod diameter & $1 \mathrm{~mm}$ & $2 \mathrm{~mm}$ & $3 \mathrm{~mm}$ & $4 \mathrm{~mm}$ & $5 \mathrm{~mm}$ \\
\hline ClearPET (RC) & 0.11 & 0.21 & 0.42 & 0.73 & 0.89 \\
\hline ClearPET (\%STD) & $35.6 \%$ & $17.2 \%$ & $18.9 \%$ & $14.2 \%$ & $13.3 \%$ \\
\hline rPET-1 (RC) & 0.14 & 0.46 & 0.66 & 0.76 & 0.81 \\
\hline rPET-1 (\%STD) & $30.8 \%$ & $18.5 \%$ & $14.4 \%$ & $14.0 \%$ & $13.1 \%$ \\
\hline
\end{tabular}
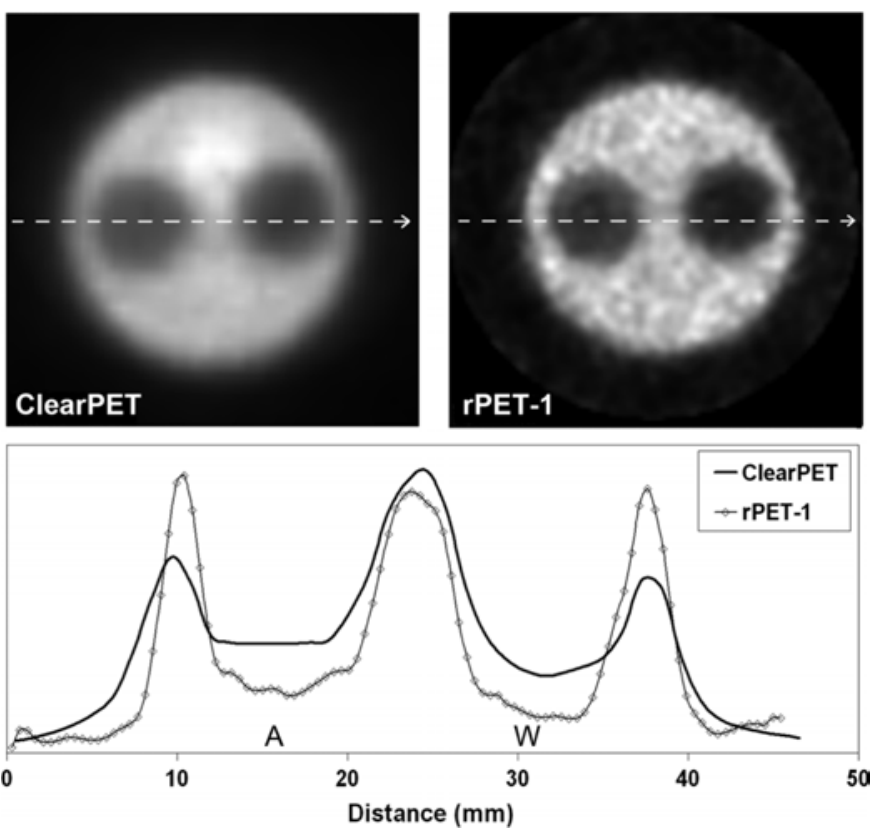

Fig. 5. Image quality phantom. Cross section (top) and activity profiles (bottom) along the air-filled region (A) and the water-filled region (W). The dotted lines show the position of the profiles.

\section{Image Quality}

The RCs and the \%STDs obtained for each of the five rods of the NEMA image quality phantom are shown in Table VI. The rPET- 1 presented a lower \%STD (6.9\%) than the ClearPET $(10.9 \%)$ in the uniform region of the phantom.

Fig. 5 shows a cross section of the cold regions in the image quality phantom for both systems. An activity profile along the cold regions is also presented. These regions were considered to measure the SOR and the noise (\%STD), which are summarized in Table VII. Since scatter correction was not applied, these values are indicative of the effect of scattered radiation on the reconstructed images.

\section{E. Derenzo Phantom Images}

The Derenzo images presented were reconstructed using 3D-OSEM algorithms in both cases. Fig. 6 shows the cross sections obtained by summing the 10 central slices of the reconstructed volumes. For each image, the window level was set in such a way that pixel values $\geq 60 \%$ of the maximum were represented as the maximum brightness. The smallest rods of 
TABLE VII

SOR AND \%STD MEASURED IN COLD REGIONS

\begin{tabular}{ccc}
\hline \hline Region & Water-filled & Air-filled \\
\hline ClearPET & $0.27,13.9 \%$ & $0.37,13.0 \%$ \\
rPET-1 & $0.15,15.4 \%$ & $0.24,15.4 \%$ \\
\hline \hline
\end{tabular}
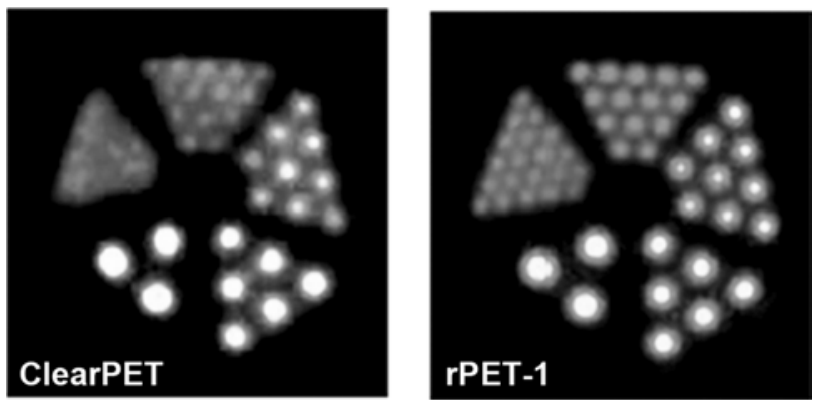

Fig. 6. Derenzo phantom scanned on the ClearPET and the rPET-1. Transaxial view, summed over 10 slices, after iterative reconstruction.

the phantom are separated $1.2 \mathrm{~mm}$ and can only be resolved for the rPET-1.

\section{SUMmary AND CONCLUSIONS}

In this work, we presented a performance evaluation of two scanners following the new NEMA NU 4-2008 protocol for small-animal PET systems. One of the main measurements of interest is the spatial resolution of the scanner. We observed that, except at the centre of FOV, rPET-1 shows an approximately $0.5-\mathrm{mm}$ smaller FWHM in the transaxial directions; this is mainly related to the smaller crystal cross-section in the rPET-1 $(1.4 \mathrm{~mm} \times 1.4 \mathrm{~mm})$ than in the ClearPET $(2.0 \mathrm{~mm} \times 2.0$ $\mathrm{mm})$. In addition, both scanners showed similar degradation of spatial resolution across the transverse axis $(\sim 1 \mathrm{~mm})$. In order to maintain this undesired loss of resolution within reasonable limits, both manufacturers implement different solutions. ClearPET uses two layers of crystals (phoswich configuration) to assess the depth of interaction (DOI) on the detectors, thus minimizing the parallax error. In contrast, rPET-1 uses planar opposite detectors covering the whole transaxial FOV, so that a high percentage of the incoming photons have parallel trajectories within the crystals. With regard to radial spatial resolution, rPET-1 shows a decreasing value from the centre towards the periphery of the FOV. This behaviour is unlike that of the ClearPET, whose detectors have a ring-type geometry. In the planar detector geometry, the lines of response that intersect crystals obliquely pass mainly through the centre, whereas at the periphery, they are mostly parallel to the crystals. Therefore, the contribution of the parallax effect to radial resolution is higher at the centre of the FOV than at the edge.

Absolute sensitivity at the centre of the FOV is five times higher for the ClearPET, as expected from the different dimensions and the use of almost 10 times higher active scintillator volume than the rPET-1. For the NEMA mouse-like phantom, the measured scatter fraction was $31.0 \%$ in the ClearPET and $24.2 \%$ in the rPET-1. With this same phantom, the peak NEC rate reached $73.4 \mathrm{kcps}$ at $0.51 \mathrm{MBq} / \mathrm{mL}$ in the ClearPET and $29.2 \mathrm{kcps}$ at $1.35 \mathrm{MBq} / \mathrm{mL}$ in the rPET- 1 . The lower sensitivity of the rPET-1 contributes to the fact that the scanner reaches its peak with more activity in the phantom.

The RCs measured ranged from 0.11 (35.6\%STD) to 0.89 $(13.3 \% \mathrm{STD})$ in the ClearPET and from 0.14 (30.8\%STD) to 0.81 (13.1\%STD) in the rPET-1. Lower SOR values were obtained for rPET-1. The smallest rods of the Derenzo phantom can only be resolved in the rPET-1 image. Nevertheless, it is important to remember that we are comparing images reconstructed with approximately the same number of counts, even though the different sensitivity of the systems makes the acquisition time almost five times longer on the rPET-1 than on the ClearPET.

\section{REFERENCES}

[1] G. D. Hutchins, M. A. Miller, V. C. Soon, and T. Receveur, "Small animal PET imaging," Inst. Lab. Animal Res. J., vol. 49, no. 1, pp. $54-65,2008$

[2] S. R. Cherry and S. S. Gambhir, "Use of positron emission tomography in animal research," Inst. Lab. Animal Res. J., vol. 42, pp. 219-232, 2001.

[3] T. J. McCarthy, "Positron emission tomography imaging as a key enabling technology in drug development," in Proc. Ernst Schering Res. Found. Workshop, 2007, vol. 62, no. 3, pp. 29-39.

[4] S. Weber and A. Bauer, "Small animal PET: Aspects of performance assessment," Eur. J. Nucl. Med. Mol. Imag., vol. 31, pp. 1545-1555, 2004

[5] J. B. Mosset et al., "Development of an optimised LSO/LuYAP phoswich detector head for the ClearPET camera," in Proc. IEEE Nuclear Science Symp. and Medical Imaging Conf. Rec., Oct. 2005, vol. 3, pp. 1641-1644.

[6] J. J. Vaquero, E. Lage, L. Ricon, M. Abella, E. Vicente, and M. Desco, "rPET detectors design and data processing," in Proc. IEEE Nuclear Science Symp. and Medical Imaging Conf. Rec., Oct. 2005, vol. 5, pp. 2885-2889.

[7] P. S. Roldan, M. Canadas, O. Dietzel, C. Pautrot, I. Sarasola, and A. Wagner, "Performance evaluation of Raytest ClearPET, a PET scanner for small and medium size animals," in Proc. IEEE Nuclear Science Symp. and Medical Imaging Conf. Rec., Oct. 2007, vol. 4, pp. 2859-2864.

[8] E. Vicente et al., "[Characterization of the animal scanner rPET]," in Proc. 24th Annu. Congress of the Spanish Society of Biomedical Engineering, 2006, (Spanish).

[9] Performance Measurements for Small Animal Positron Emission Tomographs (PETs), Standards Publication NU 4-2008, National Electrical Manufacturers Association, Rosslyn, VA, 2008 [Online]. Available: http://www.nema.org/stds/nu4.cfm

[10] Q. Bao, D. Newport, M. Chen, D. B. Stout, and A. F. Chatziioannou, "Performance evaluation of the inveon dedicated PET preclinical tomograph based on the NEMA NU-4 standards," J. Nucl. Med., vol. 50, pp. 401-408, 2009.

[11] C. Wietholt, I. Hsiao, K. Lin, Y. Chung, C. Chen, and T. Yen, "Performance evaluation of small animal PET system using NEMA standards," J. Nucl. Med., vol. 49, p. 119, 2008, (abstract).

[12] W. Luo, E. Anashkin, and C. G. Matthews, "Performance evaluation of a PEM scanner using the NEMA NU 4-2008 small animal PET standards," IEEE Trans. Nucl. Sci., vol. 57, no. 1, pp. 94-103, Feb. 2010.

[13] E. Lage et al., "Design and performance evaluation of a coplanar multimodality scanner for rodent imaging," Phys. Med. Biol., vol. 54, pp. 5427-5441, 2009.

[14] U. Heinrichs, U. Pietrzyk, and K. Ziemons, "Design optimization of the PMT-ClearPET prototypes based on simulation with GEANT3," IEEE Trans. Nucl. Sci., vol. 50, no. 5, pp. 1428-1432, Oct. 2003.

[15] H. M. Hudson and R. S. Larkin, "Accelerated image reconstruction using ordered subsets of projection data," IEEE Trans. Med. Imag., vol. 13, pp. 601-609, 2004.

[16] M. Streun, G. Brandenburg, H. Larue, C. Parl, and K. Ziemons, "The data acquisition system of ClearPET Neuro-A small animal PET scanner," IEEE Trans. Nucl. Sci., vol. 53, no. 3, pp. 700-703, Jun. 2006. 
[17] P. E. Kinahan and J. G. Rogers, "Analytic 3D image reconstruction using all detected events," IEEE Trans. Nucl. Sci., vol. 36, no. 1, pp. 964-968, Feb. 1989.

[18] K. Thielemans, S. Mustafovic, and C. Tsoumpas, "STIR: Software for Tomographic Image Reconstruction Release 2," in Proc. IEEE Nuclear Science Symp. and Medical Imaging Conf. Rec., 2006, vol. 4, pp. 2174-2176.

[19] M. E. Daube-Witherspoon and G. Muehllehner, "Treatment of axial data in threedimensional PET," J. Nucl. Med., vol. 28, pp. 1717-1724, 1987.

[20] S. C. Strother, M. E. Casey, and E. J. Hoffman, "Measuring PET scanner sensitivity: Relating countrates to image signal-to-noise ratios using noise equivalent counts," IEEE Trans. Nucl. Sci., vol. 37, no. 2, pp. 783-788, Apr. 1990.
[21] S. Yamamoto, H. Horii, M. Hurutani, K. Matsumoto, and M. Senda, "Investigation of single, random, and true counts from natural radioactivity in LSO-based clinical PET," Ann. Nucl. Med., vol. 19, pp. 109-114, 2005

[22] C. C. Watson, M. E. Casey, L. Eriksson, T. Mulnix, D. Adams, and B. Bendriem, "NEMA NU 2 performance tests for scanners with intrinsic radioactivity," J. Nucl. Med., vol. 45, no. 5, pp. 822-826, May 2004

[23] J. L. Herraiz, S. España, J. J. Vaquero, M. Desco, and J. M. Udías, "FIRST: Fast iterative reconstruction software for (PET) tomography," Phys. Med. Biol., vol. 51, pp. 4547-4565, 2006.

[24] S. Weber, B. Gundlich, and M. Khodaverdi, "Normalization factors for the ClearPET neuro," in Proc. IEEE Nuclear Science Symp. and Medical Imaging Conf., Oct. 2005, vol. 5, pp. 2632-2635. 